\title{
Ensemble forecasting with a stochastic convective parametrization based on equilibrium statistics
}

\author{
P. Groenemeijer ${ }^{1, *}$ and G. C. Craig ${ }^{1}$ \\ ${ }^{1}$ Ludwig-Maximilians-Universität, München, Germany \\ * currently at: European Severe Storms Laboratory, Wessling, Germany
}

Correspondence to: G. C. Craig (george.craig@lmu.de)

Received: 21 September 2011 - Published in Atmos. Chem. Phys. Discuss.: 11 November 2011

Revised: 4 April 2012 - Accepted: 27 April 2012 - Published: 24 May 2012

\begin{abstract}
The stochastic Plant-Craig scheme for deep convection was implemented in the COSMO mesoscale model and used for ensemble forecasting. Ensembles consisting of 100 48-h forecasts at $7 \mathrm{~km}$ horizontal resolution were generated for a $2000 \times 2000 \mathrm{~km}$ domain covering central Europe. Forecasts were made for seven case studies characterized by different large-scale meteorological environments. Each 100 member ensemble consisted of 10 groups of 10 members, with each group driven by boundary and initial conditions from a selected member from the global ECMWF Ensemble Prediction System. The precipitation variability within and among these groups of members was computed, and it was found that the relative contribution to the ensemble variance introduced by the stochastic convection scheme was substantial, amounting to as much as $76 \%$ of the total variance in the ensemble in one of the studied cases. The impact of the scheme was not confined to the grid scale, and typically contributed $25-50 \%$ of the total variance even after the precipitation fields had been smoothed to a resolution of $35 \mathrm{~km}$. The variability of precipitation introduced by the scheme was approximately proportional to the total amount of convection that occurred, while the variability due to large-scale conditions changed from case to case, being highest in cases exhibiting strong mid-tropospheric flow and pronounced mesoto synoptic scale vorticity extrema. The stochastic scheme was thus found to be an important source of variability in precipitation cases of weak large-scale flow lacking strong vorticity extrema, but high convective activity.
\end{abstract}

\section{Introduction}

A given state of the atmosphere cannot be represented perfectly in a numerical model for at least two reasons. The first reason is that for practical reasons the atmospheric state cannot be known with infinite accuracy at every location. The second reason is that a numerical model resolves the real state only up to a given level of detail, limited by the gridspacing or spectral truncation of the model, and by the time step of the simulation (Palmer, 2001). Both of these imperfections lead to errors, some of which may grow quickly over time because of the chaotic nature of the atmosphere (Lorenz, 1963). In order to obtain information on the possible future states of the atmosphere, ensemble forecasting techniques are used: multiple integrations of the model that, by introducing small perturbations, try to account for these imperfections.

First, uncertainties in the initial state of the model are accounted for by starting the model integrations with slightly modified initial conditions (Toth and Kalnay, 1993; Houtekamer and Derome, 1995; Molteni et al., 1996). Second, the problem of the error caused by the limited resolution can be addressed by using stochastic parametrizations. Parametrizations, in the context of numerical weather prediction (NWP), are algorithms that represent the effects of unresolved processes such as radiation, boundary-layer processes and convection. In contrast to traditional deterministic parametrizations, the fundamental idea of stochastic parametrizations is that the small-scale processes that they represent are not uniquely determined by the resolved flow, but are also affected by unresolved processes that are inherently unknown but can be modelled in a stochastic manner (Palmer, 2001). 
Several approaches to stochastic parametrization have been taken, typically with the aim to address sources of uncertainty, that, when taken into account, can allow ensemble spread to grow without sacrificing accuracy. An important advantage of stochastic schemes is that they introduce variability throughout the integration, which helps to generate sufficient ensemble spread later in the simulation without having to introduce unrealistically large perturbations to the initial state. Buizza et al. (1999) introduced randomness by multiplying the effects of all parametrized processes by a factor between 0.5 and 1.5 , and have shown that this increased the spread of the ensemble, and improved the skill of the probabilistic prediction of weather parameters such as precipitation. Shutts (2005) introduced stochastic perturbations to backscatter a portion of the kinetic energy that is normally dissipated by parametrized processes, resulting in an improvement of probabilistic measures of forecast skill. Other parametrizations based on this concept have been tested by Bowler et al. (2009), Berner et al. (2009) and Tennant et al. (2011). Berner et al. (2009) found that stochastic kinetic energy backscatter leads to improved rainfall forecasts in the ECMWF ensemble forecast system, and Bowler (2009) and Tennant et al. (2011) reported an improvement of the growth rate of the ensemble spread of some variables, and an improved forecast skill at short lead times.

Many stochastic parametrizations have focused on cumulus convection, which shows substantial variability on scales close to the truncation limit of current numerical models. Lin and Neelin (2003) developed two stochastic schemes for deep convection, one by making the closure time-scale (the time in which destabilization is compensated by convective processes) stochastic, the other by adding stochastic perturbations to the vertical distribution of the temperature tendencies produced by the scheme. These helped to increase variability, and excite low wavenumber and -frequency disturbances, repectively. Bright and Mullen (2002) developed a stochastic version of the Kain-Fritsch convective scheme by adding a stochastic component to its trigger function, resulting in a slight increase in skill and ensemble spread. Teixeira and Reynolds (2008) added stochastic variability to a cumulus parameterization under the assumption that the standard deviation of the fluctuations was proportional to the tendency, and found that it produced substantial variability, particularly in the tropics, leading to increased ensemble spread both in the tropics and extra-tropics.

In each of these studies, the spatial and/or temporal autocorrelation of the perturbations were controlled by parameters that could be adjusted to produce a desired increase in ensemble spread. In contrast, Berner et al. (2008) employed a cellular automaton to generate patterns of spatial variability, while Khouider et al. (2003) showed how a birth-death model of convective initiation could be used to formally derive a stochastic parametrization for larger scales. In the scheme developed by Plant and Craig (2008, hereafter PC08), used in this study, the convective variability is constrained by an underlying physical theory, which is based on equilibrium statistics (Craig and Cohen, 2006). In this study we report on the use of the PC08 scheme, previously developed and tested only in a single-column mode, in a limited area model.

If the goal of using a stochastic parametrization scheme, like the PC08 scheme, the variability it produces must be quantified and compared with other sources of variability, such as perturbations in the initial conditions. If the stochastic scheme is to have a sizeable effect on the ensemble spread, the amount of introduced variability must not be negligible in comparison to those other sources. One may anticipate that the relative importance of the stochastic variability is dependent on the meteorological situation, because both the overall magnitude and the degree of randomness of the parametrization are likely to be weather dependent (Craig and Cohen, 2006).

The variability that a stochastic parametrization scheme introduces can be split into two parts: a direct and an indirect component. The direct component is the variability that develops instantaneously because of different stochastic microscopic realizations in the same resolved (macroscopic) flow. This component has the same spatial and temporal scales as the tendencies provided by the stochastic scheme. In contrast, the indirect component is variability of the resolved flow that develops because of upscale growth of the direct variability (Tan et al., 2004; Zhang et al., 2007). This difference is of importance, because the direct component of variability and the corresponding probabilistic information may alternatively be arrived at by statistical post-processing of a deterministic forecast, as done by e.g. Applequist et al. (2002), Bremnes (2003) or Theis et al. (2005). In contrast, such post-processing cannot account for the impact of smallscale variability on the large-scale flow, so that the stochastic parametrization approach has the potential to be qualitatively superior to such techniques.

Based on the above considerations, our evaluation of the performance of the PC08 parametrization is directed towards the following questions:

1. How large is the impact of the stochastic variability introduced by the convective scheme compared with that introduced by varying initial and boundary conditions?

2. How much of the variability can be considered a different realization of the same resolved flow, and how much is due to changes of the resolved flow due to upscale error growth?

3. How does the variability depend on the weather pattern?

This article is structured as follows. Section 2 describes the implementation of the Plant-Craig scheme in the limited-area model. In Sect. 3, the set-up of the ensemble forecasting system is presented. The results are presented and discussed in Sect. 4, and we formulate our conclusions in Sect. 5. 


\section{Implementation of the Plant-Craig scheme in the COSMO model}

For the current study, the PC-scheme (PC08) was integrated into the COSMO (Consortium for Small-scale Modelling) model (Schättler et al., 2007), version 4.8. This model is developed and used by the Consortium for Small Scale Modelling (COSMO), and is both non-hydrostatic and fully compressible. It is used, for example, at the German Weather Service (Deutscher Wetterdienst, DWD) in daily forecasting operations, and for mesoscale meteorological studies. The model uses a terrain-following coordinate system. Its computational grid is an Arakawa C-grid with vertical Lorenz grid staggering.

The PC-scheme produces small-scale variability while maintaining quasi-equilibrium on a large scale. Large-scale fields are obtained by applying spatial or spatio-temporal averaging, and a CAPE-based closure is assumed. To obtain the small-scale variability, the scheme draws convective elements, herein interchangeably called plumes or clouds, from a probability density function (Craig and Cohen, 2006), normalized with this closure. The feedback of each plume on the resolved flow is calculated with an adapted version of the Kain-Fritsch plume model (Kain and Fritsch, 1990). For a comprehensive description of the PC-scheme, the reader is referred to Plant and Craig (2008). In what follows, we will recall its main features and describe a number of modifications that were made upon its implementation in the COSMO model.

The PC-scheme is called from the model for each $(\mathrm{x}, \mathrm{y})$ grid cell every convective time step, to provide the convective feedback. In the current study the convective time step was set to 10 model time steps (5 minutes and $30 \mathrm{~s}$, respectively).

Figure 1 summarizes the different steps carried out during a single call of the scheme. First, plumes that were activated in earlier call to the scheme and have exceeded their lifetime are removed (Fig. 1). As in PC08, the lifetime was set to 45 minutes regardless of plume properties. Next, the scheme evaluates whether any layers could act as source layers for new convective plumes, and calculates the closure mass flux (Fig. 1). To do so, the PC-scheme uses vertical profiles of temperature and humidity that represent an averaged atmospheric state. This averaging must be over a sufficient area (or time period) to contain a large number of clouds, to ensure that the near-equilibrium statistics on which the scheme is based are valid.

Ideally, as in $\mathrm{PC} 08$, the determination of the size of this area would consist of an iterative process, whereby (i) an arbitrary initial cloud spacing is chosen, (ii) an averaging of the atmospheric state over an area containing many clouds is performed, and (iii) a cloud density is computed by the scheme and used to give a better estimate of the cloud spacing for the next iteration. However, it is not evident that such a procedure would always converge, and it is computationally expensive. Therefore, we have chosen to use a fixed av-

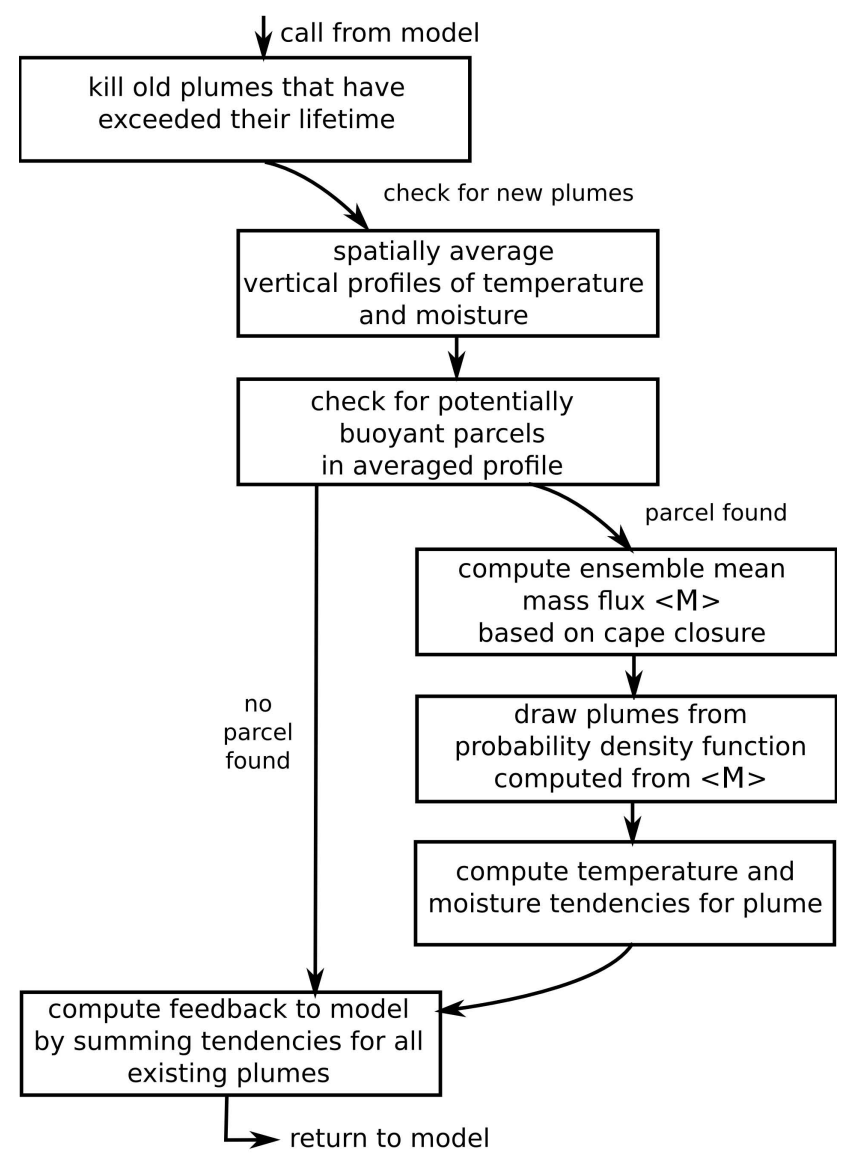

Fig. 1. Flowchart displaying the tasks performed in the Plant-Craig convection scheme.

eraging area of about $1200 \mathrm{~km}^{2}$ or 25 grid points which was the largest area that could be implemented given the technical constraints of the model. Since about 10 to 100 clouds typically occur within an area of this size in regions of active convection, the prerequisite that the mass flux be balanced "over a large ensemble of clouds" was only marginally met.

Using the averaged profile, the scheme attempts to launch plumes from a series of layers (as described in PC08, their Sect 3.2), evaluating whether a lifted parcel would be positively buoyant, thereby initiating a convective plume. In PC08 the temperature of each lifted parcel was initially increased by $0.2 \mathrm{~K}$ and, if it did not become buoyant, increased several times by $0.1 \mathrm{~K}$. To reduce computational cost, we have instead applied a single $1.0 \mathrm{~K}$ perturbation. If a parcel with this perturbation would not become buoyant, the layer was discarded as a potential source for convection.

If a buoyant parcel is found (right panels of Fig. 1), a closure mass flux $\langle M\rangle$ is calculated, that produces the stabilization required to offset $90 \%$ of the CAPE with a fixed closure timescale $\tau_{\mathrm{c}}$. For this calculation, the plume model of the Kain-Fritsch scheme (Kain and Fritsch, 1990; Kain, 2004) is used. Using this value, a probability density function 
Table 1. List of selected cases.

\begin{tabular}{llcl}
\hline Case & $\begin{array}{l}\text { Initial date and time } \\
\text { (yyyy mm dd hh) }\end{array}$ & $\begin{array}{l}\text { Domain-averaged 48 } \\
\text { convective precipitation }(\mathrm{mm})\end{array}$ & Description \\
\hline S1 & 2005010612 & 0.12 & Winter Storm Erwin/Gudrun \\
W1 & 2006071112 & 1.84 & PRINCE field campaign, Groenemeijer et al. (2009) \\
M1 & 2007071912 & 1.16 & COPS field campaign IOP 9c, Corsmeier et al. (2011) \\
M2 & 2008062412 & 1.08 & Strong squall line in central parts of Europe, \\
& & 1.09 & Púčik et al. (2011); Simon et al. (2011) \\
M3 & 2008091112 & 2.09 & Mediterranean cyclone case, Grams et al. (2011) \\
W2 & 2009063012 & 0.48 & Diurnally-driven convection case \\
W3 & 2010062612 & & Summer day with little convective activity \\
\hline
\end{tabular}

(PDF) is defined that expresses the chance that a cloud with a certain radius $r$, which is assumed to be related to the mass flux $m$ according to $m=\left(<m>/<r^{2}>\right) r^{2}$, is launched within the time interval $\delta t$ between two calls of the convection scheme. Angle brackets denote an average over the theoretical ensemble of convective clouds. This PDF may be expressed as

$p(r) d r=\frac{2 r}{\left\langle r^{2}\right\rangle} \exp \left(\frac{-r^{2}}{\left\langle r^{2}\right\rangle}\right) d r$

The scheme considers a range of $r$ values from 0 to $3<$ $r^{2}>^{1 / 2}$ and, for each radius, determines, whether a cloud will be launched by comparing a pseudo-random number against the value of $p(r)$ for that radius.

During test simulations, it was found that with the values of mass flux, mean cloud radius and closure timescale used in $\mathrm{PC} 08$, too little convective precipitation was produced in the simulations. A combination of the following adjustments enabled the scheme to produce amounts of convective precipitation comparable to the non-stochastic Kain-Fritsch (1990) and Tiedtke (1989) schemes, and are explained in detail below:

1. the root mean squared cloud radius $<r^{2}>^{1 / 2}$ was increased from $450 \mathrm{~m}$ to $1200 \mathrm{~m}$

2. the closure time scale $\tau_{\mathrm{c}}$ was fixed and set to $600 \mathrm{~s}$, or $10 \mathrm{~min}$.

3. the mean mass flux per cloud, $<m>$, was reduced from $2 \times 10^{7} \mathrm{~kg} \mathrm{~s}^{-1}$ to $1 \times 10^{7} \mathrm{~kg} \mathrm{~s}^{-1}$

The increase of the mean squared cloud radius increased the convective activity very strongly. This can be understood since PC08 calibrated their values to produce results similar to that of a cloud-resolving simulation over a tropical ocean, where it is known that the boundary layer is typically shallower than in mid-latitudes with which we are concerned here, leading to correspondingly narrower updraughts. Moreover, entrainment is probably stronger and more inhibiting for small convective plumes in the mid-latitude troposphere than it is in the tropics. A refinement of the scheme in future work could be to vary the mean cloud radius depending on boundary layer height or the lifting condensation level, perhaps among other predictors of cloud radius. After changing the mean cloud radius, it was still found that a very large fraction of the rainfall produced in the simulations originated from the grid-scale rather than the PC convective scheme. It appeared that the parametrized convection was not able to reduce the CAPE rapidly enough to prevent grid-scale convection from developing. We hypothesize that this is the case because the gravity waves that communicate the stabilization in the real atmosphere (Bretherton and Smolarkiewicz, 1989) are strongly damped by numerical diffusion, so that temperature perturbations are overly confined to the grid cell where a plume occurs. A solution to this problem would be to parametrize the feedback of the scheme in such a way that the stabilization occurs not only in the grid cell where the convection occurs, but also in neighbouring grid cells. A quicker solution, which we have chosen, was to reduce the closure time-scale $\tau_{\mathrm{c}}$, making convection more intense and more likely to set in before explicit convection sets in. The final adjustment of reducing $\langle m\rangle$ by $50 \%$ also increased the tendency for the scheme to produce convection, since this favours larger numbers of plumes.

After launching a new plume, the temperature and moisture tendencies that it produces are calculated. Finally, for all plumes, both newly launched and pre-existing, the sum of their effects on the vertical temperature and moisture profiles is computed and returned to the model.

\section{Ensemble forecasting experiments}

The ensemble experiment was structured as follows. We used the European Centre for Medium-Range Weather Forecasts (ECMWF) Ensemble Prediction System (EPS), consisting of 50 members per forecast. These forecasts were re-run from ECMWF operational analyses out to $48 \mathrm{~h} .10$ members of the ensemble forecast were selected using the RM (representative member) selection algorithm developed by Molteni et al. (2001). This algorithm involves a cluster 


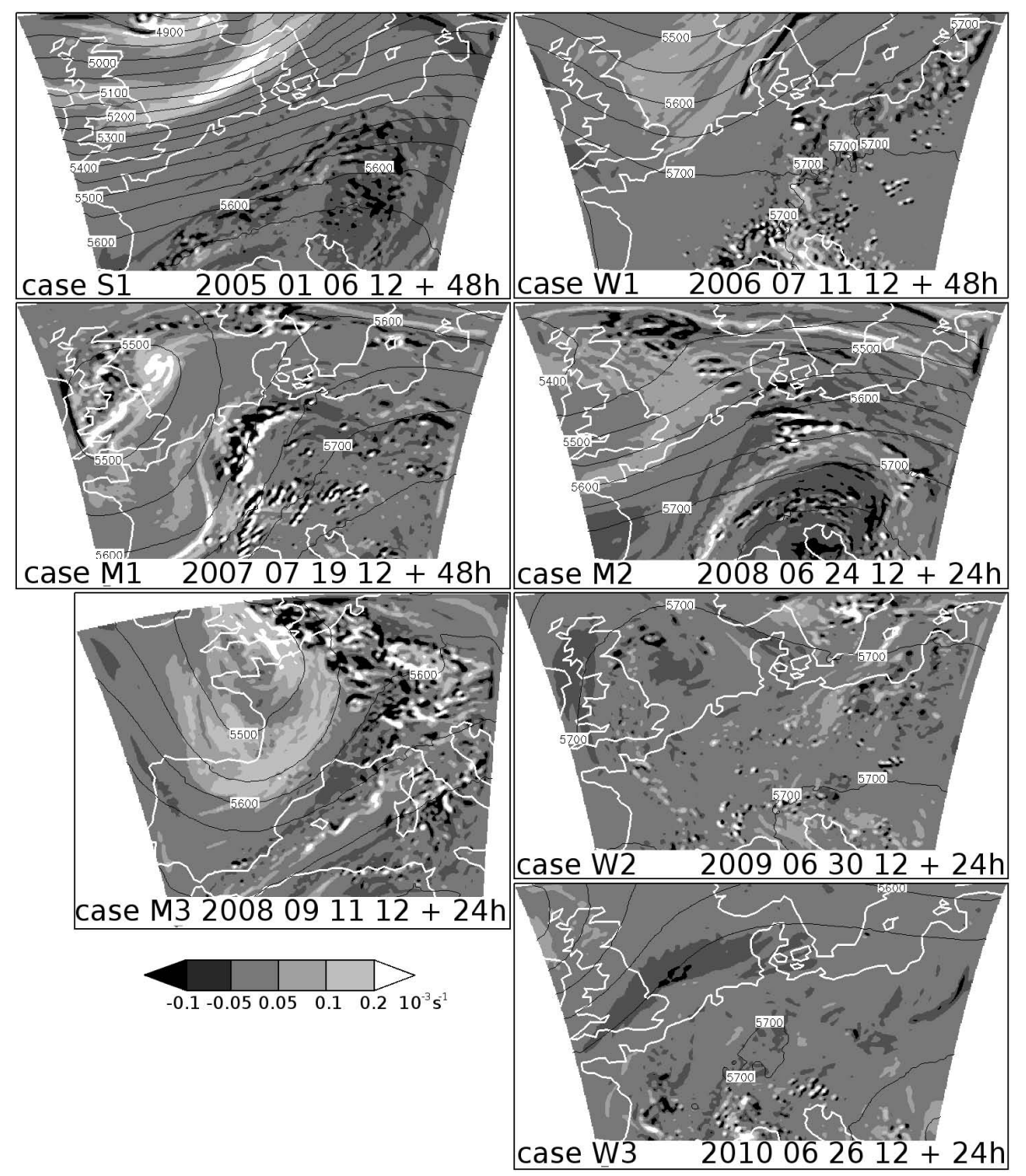

Fig. 2. $500 \mathrm{hPa}$ relative vorticity $\left(\mathrm{s}^{-1}\right.$, shaded), and geopotential height (m, in black contours) for selected cases.

analysis followed by the selection of a representative member from each of the clusters. These representative members were used as initial and boundary conditions for the simulations with the COSMO model. For each EPS member 10 COSMO simulations were carried out, using different random seeds for the stochastic parametrization, resulting in a 100 member ensemble of COSMO forecasts for each case.

The COSMO model was set up following the COSMO-EU configuration used by the German Weather Service to support operational weather forecasting (DWD, 2011), the only differences being the use of the PC-scheme for convection instead of the Tiedtke scheme, and the use of a different domain. The domain of the simulations for our study stretched across central Europe (Fig. 2) and had a size of approximately $2100 \times 2100 \mathrm{~km}$. For one case (M3) the domain was shifted southwards to better capture the Mediterranean cyclone that was responsible for most of the precipitation The grid-spacing was $0.0625^{\circ}(\sim 7 \mathrm{~km})$ and the simulations were run out to $48 \mathrm{~h}$.

Seven cases were selected for this study (see Table 1) in an attempt to represent situations with different amounts of large-scale forcing and convective activity. Moreover, five of the cases have been the subject of prior scientific studies. As an indication of large-scale forcing the presence of a largescale trough, characterized by a local maximum of vorticity at $500 \mathrm{hPa}$, was used. Each of the cases was subjectively assigned to one of three categories: W (weakly-forced), M (moderately-forced), or S (strongly-forced).

As an example of a strongly-forced case, Fig. 2 (left top) shows the relative vorticity and geopotential of case $\mathrm{S} 1$, which features a maximum of relative vorticity over the northwestern part of the domain which is embedded in a strong southwesterly-flow, indicative of strong advection of cyclonic vorticity. According to quasi-geostrophic theory, this implies forcing for upward vertical motion. Cases 

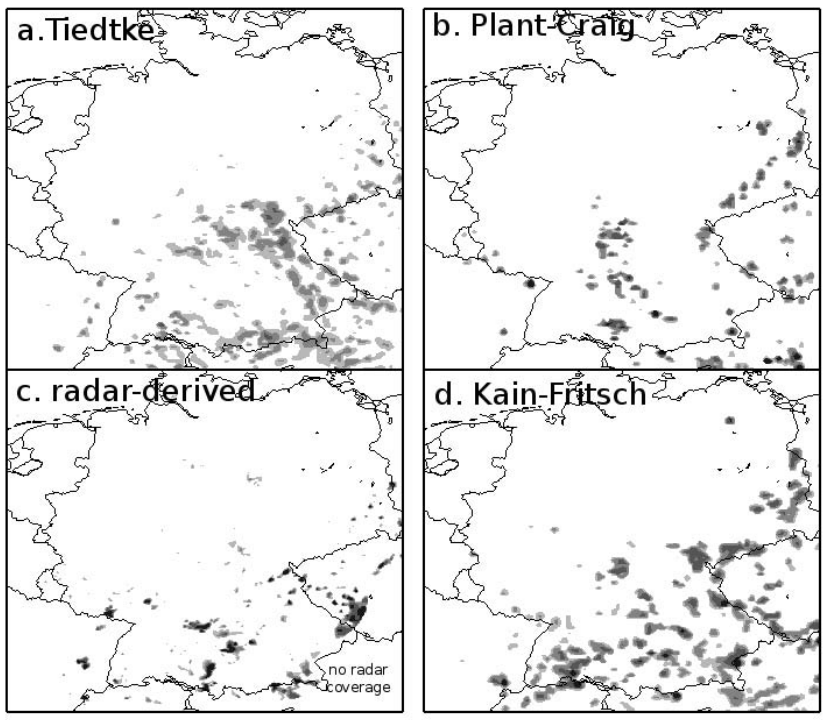

12 July 200600 UTC $+16 \mathrm{~h}$
0.3 $\begin{array}{llll}1.0 & 3.0 & 10.0 \mathrm{~mm} / \mathrm{h}\end{array}$

Fig. 3. Comparison of precipitation rates of the Tiedtke (a), PlantCraig (b) and Kain-Fritsch (d) convective schemes, and the radarderived rates (c) $16 \mathrm{~h}$ after initialization.

categorized as moderately-forced (M1, M2, and M3) feature local maxima of relative vorticity as well (for example, for case M1, a feature stretching over eastern France), but those are weaker and embedded within a weaker steering flow. It must be noted that strong small-scale maxima and minima of vorticity coinciding with convective activity can be seen in all but the $\mathrm{S} 1$ case. These, however, have been ignored for the categorization of the cases, as they are much smaller than the Rossby radius of deformation and hence have little meaning in the context of quasi-geostrophic dynamics. Finally, for cases categorized as weakly forced, large-scale vorticity maxima were absent (W2, W3) or very weak (W1).

\section{Results and discussion}

\subsection{Structure of the PC-scheme precipitation field}

The stochastic nature of the PC-scheme is reflected in the spatial distribution of precipitation that the scheme produces. Figure 3. shows snapshots of the precipitation rate that illustrate the differences between the Tiedtke (a), Plant-Craig (b), and Kain-Fritsch (c) convective schemes. Additionally, although validation of the stochastic Plant-Craig scheme with regard to observations is not part of this study, a radar-derived estimate of the precipitation rate is provided for reference (d).

The spottiness of the PC-scheme reflects its tendency towards creating convective clouds within certain grid cells, while neighboring grid cells may not contain any convective clouds. For the grid cell size used here, there is a significant chance that the random process will produce no convection

in a cell in which forcing is present. In contrast, the Tiedtke scheme (Fig. 3a) appears to have a much smoother precipitation field, with weaker maxima, which is probably in part the result of the scheme compensating the forcing directly and immediately. The Kain-Fritsch scheme is also somewhat smoother than the PC-scheme, but the maximum rain rates are as intense. This combination results in a positive bias of the precipitation rate of the scheme. It is unclear what causes this behavior in this particular snapshot, and is not typical of all forecast hours (not shown).

\subsection{Differences among stochastic realizations}

Now we turn to differences between various runs of the PCscheme, which we will refer to as stochastic realizations. An example given in Fig. 4 displays the accumulated $48 \mathrm{~h}$ precipitation of four simulations of the W1 case. Panels (a), (b), and (c) were run with initial and boundary conditions of a particular EPS member (number 1), while Panel (d) used a different EPS member (number 2). Among the simulations driven by the same EPS member 1, the rainfall distribution differs, for example within the area marked by square " 1 ". The difference manifests itself primarily on very small scales, i.e. scales much smaller than the square (which has a size of $150 \mathrm{~km}$ by $150 \mathrm{~km}$ ), and is a direct consequence of the stochastic nature of the PC-scheme. Each of the simulations (a), (b) and (c), however, contain comparable amounts of accumulated precipitation when averaged over the entire square. This can be explained by the fact that the simulations are merely different realizations of a convective ensemble with similar macroscopic properties, including the CAPE field. The distribution of precipitation produced by simulation (d) within the square, however, is a realization of a different convective ensemble. In this particular example, the forcing for convection, as conveyed by the ensemble mean mass flux $\langle M\rangle$, was much smaller in simulation (d) than in (a), (b) and (c), resulting in much less precipitation.

In contrast to the accumulations within square " 1 ", some evidence can be found that simulations driven by the same initial and boundary conditions can occasionally develop variability on scales of the size of a convective ensemble ( $35 \mathrm{~km}$ by $35 \mathrm{~km}$ ) or larger. Within the squares labelled " 2 " in Fig. 4, one can see that simulations (a), (b), and (c) not only differ on scales much smaller than the square: square "2" in simulation (a) contains more precipitation (average: $12.2 \mathrm{~mm}$ ) than in simulations (b) and (c) $(7.8$ and $10.4 \mathrm{~mm}$, respectively). Closer inspection reveals that this was the result of an area of non-convective precipitation being larger and more intense. This area of precipitation has a spatial extent smaller than the square, but much larger than the $35 \times 35 \mathrm{~km}$ area supposed to constitute a convective ensemble. This indicates that the stochastic parametrization has had an upscale influence through interaction with the grid-scale cloud scheme 

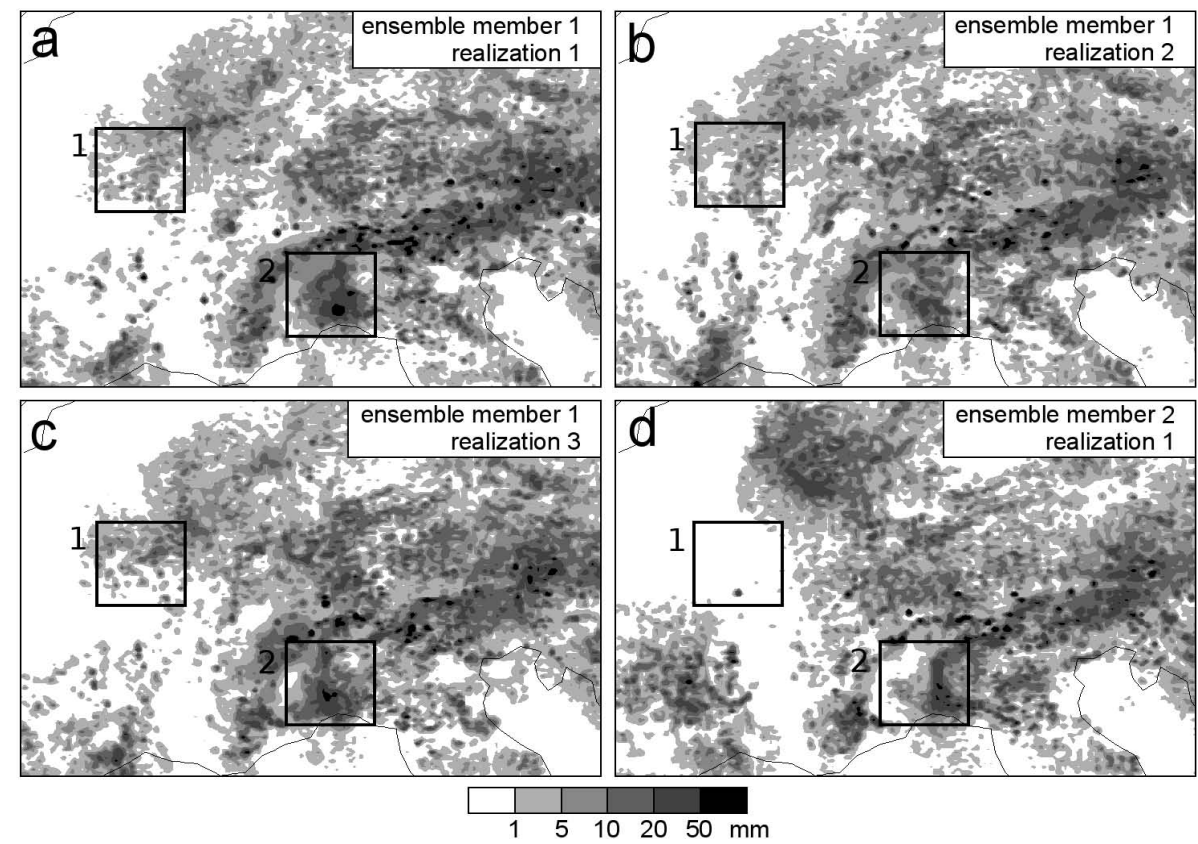

Fig. 4. 48-h accumulated rainfall of four realizations of the PC-scheme across part of the domain centered on the western Alps. Panels (a), (b) and (c) are simulations run with intial and boundary conditions provided by the same EPS ensemble member (1), panel (d) is a simulation driven by another member (2). Squares 1 and 2 denote regions discussed in the text.

\subsection{Relative impact of the stochastic perturbations}

A primary objective of the current study is to compare the variability produced by the initial and boundary conditions with that produced by the stochastic convective parametrization. In order to do this, the variance among all members is calculated for a given quantity, and compared with the average variance among members driven by the same EPS member. The latter represents the variability owing to the stochastic scheme, whereas the total variance represents variability introduced by all sources. Formally this can be written down as follows.

For a domain with $N$ gridpoints, we define $r_{i j}(x, y)$ to denote the value of $r$ (for example, the hourly rainfall accumulation) at a grid point $(x, y)$ of stochastic realization $i$ driven by EPS member $j$, where $i$ and $j$ each range from 1 to 10 . Then the mean $r$ averaged over all 10 stochastic realizations of driving member $j$ is given by

$\bar{r}_{j}(x, y)=\frac{1}{10} \sum_{i=1}^{10} r_{i j}(x, y)$,

and the mean $r$ averaged over all simulations by

$\overline{\bar{r}}(x, y)=\frac{1}{100} \sum_{j=1}^{10} \sum_{i=1}^{10} r_{i j}(x, y)$.
The sample variance over all stochastic realizations of driving member $j$ is then

$s_{j}^{2}=\frac{1}{10 N} \sum_{i=1}^{10} \sum_{x, y}\left(r_{i j}(x, y)-\bar{r}_{j}(x, y)\right)^{2}$.

The variance due to the stochastic scheme, which we will call internal variance, can be expressed as

$s_{i n t}^{2}=\frac{1}{10} \sum_{j=1}^{10} s_{j}^{2}=\frac{1}{100 N} \sum_{i=1}^{10} \sum_{j=1}^{10} \sum_{x, y}\left(r_{i j}(x, y)-\bar{r}_{j}(x, y)\right)^{2}$

and the total variance as

$s_{\mathrm{tot}}^{2}=\frac{1}{100 N} \sum_{i=1}^{10} \sum_{j=1}^{10} \sum_{x, y}\left(r_{i j}(x, y)-\overline{\bar{r}}_{j}(x, y)\right)^{2}$.

Figure 5 shows an example of the total and internal variances, taking $r$ to be 1-hourly rainfall accumulations, for one case (W1), with values shown for times at six hour intervals throughout the forecast. For this weakly forced case, the internal variability associated with the stochastic convection scheme accounts for the major part of the variance at all times. One-hour accumulated precipitation (convective and total) is also plotted in Fig. 5, and shows a clear diurnal cycle. The forecast hours $6,24,30$, and 48 , at which variability is greatest, correspond to times at which diurnallydriven convection is most intense, i.e. 18:00, 12:00, 18:00 and 12:00 UTC, respectively (12:00 UTC is just after local 


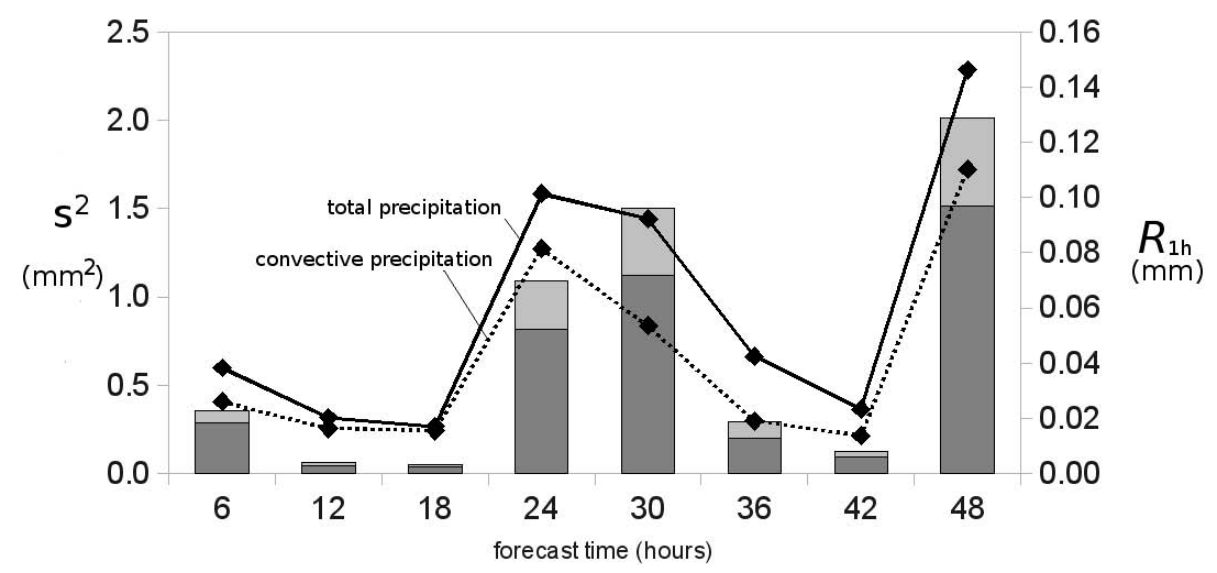

Fig. 5. Variance of 1-hourly precipitation accumulations among members driven by the same initial and boundary conditions $\left(s_{\text {int }}^{2}\right.$, internal variance; dark grey bars), and the variance among all simulations $\left(s_{\mathrm{tot}}^{2}\right.$, total variance; complete bars), and the spatially averaged 1-hourly accumulated convective (dotted line) and total (continuous line) precipitation at 8 different forecast times for case W1. The accumulations are over the hour preceding the respective forecast time.

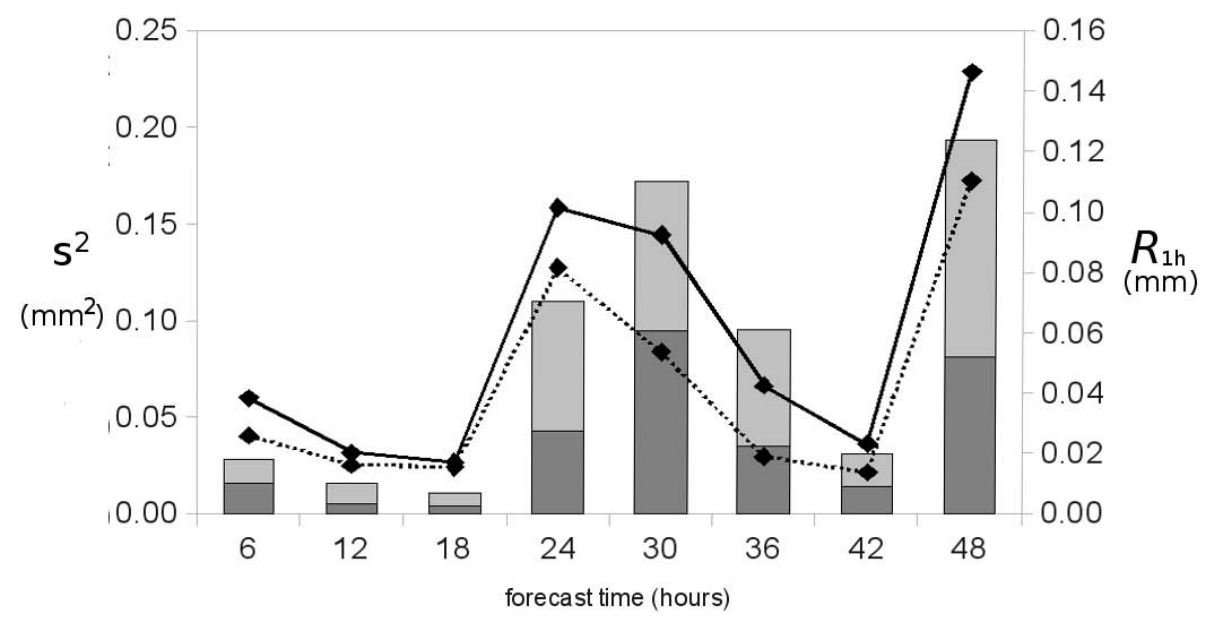

Fig. 6. As for Fig. 5 but after smoothing the precipitation field (see Text).

noon and 18:00 UTC is in the early evening). These times also show the greatest total and internal variance in the precipitation. The fraction of the total variance accounted for by the internal variance remains approximately uniform throughout the diurnal cycle, 0.74 on average.

The spotty nature of the precipitation fields produced by the PC-scheme (Fig. 3) suggests that much of the internal variance occurs on spatial scales close to the model grid length. This is confirmed in Fig. 6, which shows the total and internal variances computed from precipitation fields that have been filtered using the same 25 -point $(35 \mathrm{~km} \times 35 \mathrm{~km})$ smoothing kernel that was used to calculate the convective forcing within the PC-scheme. The total variance in the ensemble of smoothed fields is about an order of magnitude less than that of the unsmoothed fields (Fig. 5). It is significant however that even after smoothing, the internal variance is still about half as large as the total variance, showing that the PC-scheme is still a major source of ensemble spread, even on scales substantially larger than those directly forced by the stochastic variability. It is also apparent in Fig. 6 that the total and internal variances follow the diurnal cycle in precipitation, as was the case for the unsmoothed fields.

A relationship between the variance introduced by the stochastic convection and the amount of convective precipitation is expected from the design of the PC08-scheme. The equilibrium distribution function for convective mass flux corresponds to a marked Poisson process (Craig and Cohen, 2006), and has the property that the variance is proportional to the mean convective mass flux $\langle M\rangle$, i.e. $\left\langle(M-\langle M\rangle)^{2}\right\rangle=$ $2\langle M\rangle\langle m\rangle$ (Cohen, 2001). If we assume that precipitation $r$ in a grid box is proportional to the convective mass flux $M$, then a similar proportionality will hold between $r$ and its mean. To verify this, Fig. 7 shows the internal variability $s_{\text {int }}^{2}$, plotted as a function of 1-hourly convective precipitation at six hour 


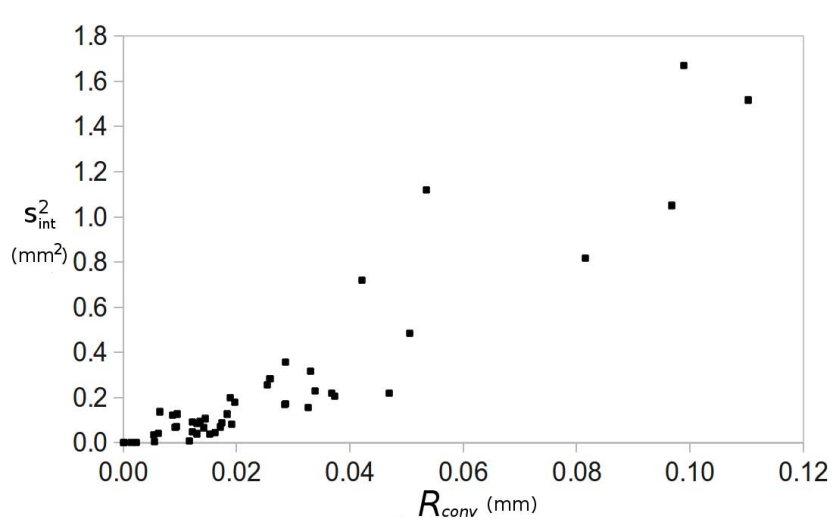

Fig. 7. The mean internal sample variance of the precipitation accumulation $s_{\text {int }}^{2}$ as a function of the mean convective precipitation accumulation $R_{\text {conv }}$, over the hour preceding the $6,12,18,24,30$, 36,42 and $48 \mathrm{~h}$ forecast times for all cases listed in Table 1.

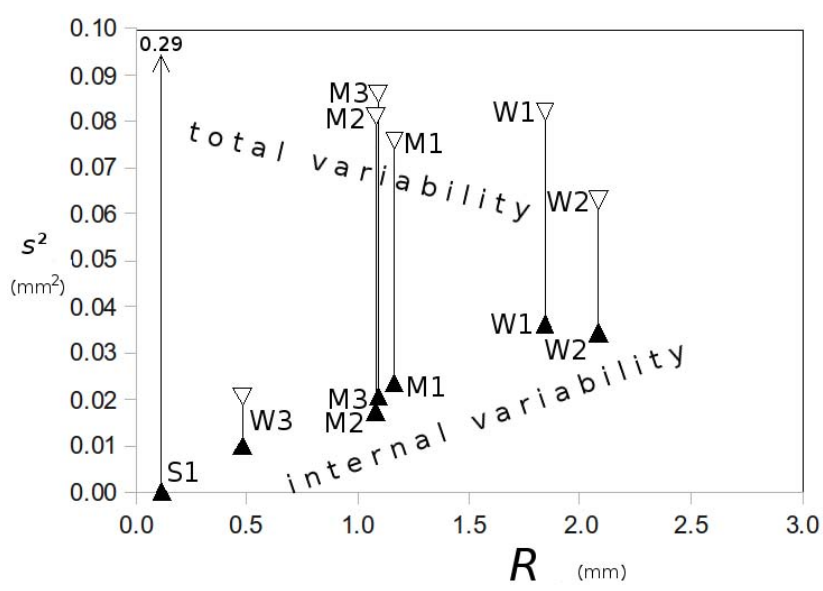

Fig. 8. Total $s_{\text {tot }}^{2}$ (open triangles), and internal $s_{\text {int }}^{2}$ (closed triangles) sample variance of the smoothed precipitation accumulations over the hour preceding the $6,12,18,24,30,36,42$, and $48 \mathrm{~h}$ forecast time, as a function of the ensemble average accumulated precipitation over the $48 \mathrm{~h}$ forecast period $R$.

intervals for all 7 cases. The relationship is indeed approximately linear, although with some scatter, which may reflect a different proportionality between mass flux and precipitation in different weather regimes.

The relationships between the total precipitation and total and internal variances are explored in Fig. 8. For clarity, the variances computed at 6-hourly intervals are averaged over the two day period of each forecast and plotted against the $48 \mathrm{~h}$ accumulated convective precipitation. For all case studies the internal variance (closed triangles) is roughly proportional to the amount of convective precipitation. The total variance however shows no such relationship. Instead it would appear that the ratio of internal to total variance depends upon the meteorological environment (Fig. 9). The cases that were subjectively categorized as weakly forced (W1, W2, W3) have ratios of internal to total variance of

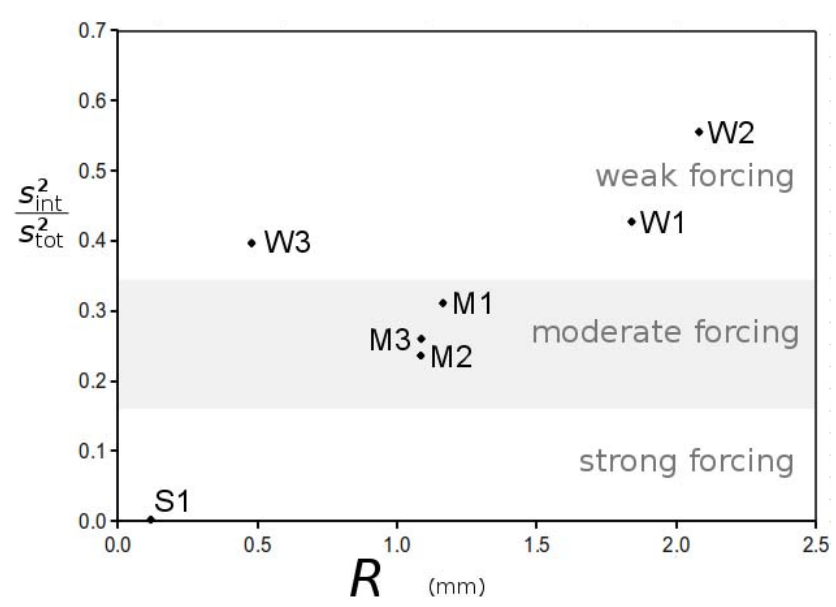

Fig. 9. Ratio of internal $\left(s_{\text {int }}^{2}\right)$ to total $\left(s_{\text {tot }}^{2}\right)$ sample variance of the smoothed precipitation accumulations over the hour preceding the $6,12,18,24,30,36,42$, and $48 \mathrm{~h}$ forecast time as a function of the ensemble average accumulated precipitation over the $48 \mathrm{~h}$ forecast period $R$.

about $0.5(0.39,0.42$ and 0.55 respectively) after smoothing. Those that had moderate forcing (M1, M2, M3) have ratios much lower than that $(0.24,0.26$, and 0.31 respectively). The strongly-forced winter storm case (S1) has a ratio of 0.02 . These results indicate that the stochastic scheme has a relatively high impact when external forcing is weak, less impact when convection is more strongly constrained by the largescale flow, and negligible impact for the winter storm case where precipitation was mainly non-convective. This result is in agreement with that of Stensrud et al. (2000), who, in a comparison of model physics ensembles with initial condition ensembles, found that model physics ensembles were more skillful when large scale forcing of upward motion is weak, and initial condition ensembles more skillful when large scale forcing of upward motion is strong. It was found that the influence of the stochastic scheme on two other variables, the $500 \mathrm{hPa}$ geopotential height and the $850 \mathrm{hPa}$ temperature, was much smaller than that on precipitation. The percentage of variance attributable to the stochastic scheme ranges from $0.042 \%$ (case S1) to $1.6 \%$ (case W2) after $48 \mathrm{~h}$ of simulation. The temperature at $850 \mathrm{hPa}$ is somewhat more sensitive to the stochastic scheme, with the percentage ranging from $0.59 \%$ (case $\mathrm{S} 1$ ) to $10.5 \%$ (case W2).

\section{Conclusions}

The impact of a stochastic convective parametrization has been investigated by running a large 100 member ensemble consisting of 10 stochastic realizations of a simulation initiated with 10 members from the ECMWF EPS, for seven individual weather situations that differ in the amount of forcing and convective activity. Based on an evaluation of the 
precipitation fields produced by the simulations, we can answer the questions posed in the introduction.

First, regarding the relative impact of the stochastic scheme we can conclude that it is, compared with the effects of varying initial and boundary conditions, relatively strong when considering hourly precipitation accumulations at the model grid scale. The fraction of sample variance attributable to the stochastic scheme varies between $1.7 \%$ (case S1) and $76.3 \%$ (case W2) of the total variance depending on the weather pattern. The variability introduced by the stochastic scheme appears to be approximately proportional to the total amount of convective precipitation.

Second, concerning the impacts of the stochastic scheme on the resolved flow, we find that smoothing the precipitation reduces the variability considerably, but a large impact due to the stochastic scheme remains in all of the simulated weather patterns (viz. dark grey bars in Fig. 6), except for the winter storm (S1). This implies that at least some of the convective variability still has an effect on larger scales. Visual inspection of selected precipitation fields (e.g. Fig. 3) shows indications of such upscale error growth, but these signals are very subtle. We assume that, given a long enough simulation, such errors will grow further upscale. To investigate this, a global simulation is needed rather than a regional one as in the present study.

Last, regarding the dependency of the importance of stochastic perturbations on the weather type, we conclude that in weather patterns that are weakly-(strongly-)forced the relative impact of the stochastic scheme is high (low). This can be explained by the fact that the total variance in weakly-forced weather patterns tended to be smaller than in the strongly-forced patterns, while the variance contributed by the stochastic scheme is proportional to the amount of convective rainfall. The relative importance of the stochastic scheme, is thus larger in the weakly-forced cases.

The results of this first study show that a physically-based stochastic parametrization of convective variability can provide a significant source of variance for ensemble forecasting of convective precipitation. In ensemble systems that employ cycling (e.g. ensemble transform or ensemble Kalman filter), the impact of the stochastic parametrization may be even greater since the initial ensemble will be influenced by stochastic perturbations from earlier times (Reynolds et al. 2008). An important next step is to verify that the increased variance resulting from the stochastic convection scheme leads to better probabilistic forecasts when compared to observations (this work is in progress).

Finally, the dependence of the convective variability on weather regime hints at a potential for developing flowdependent algorithms to generate forecast products, or adaptive ensemble design where computational resources are more optimally assigned to capture the forecast uncertainty inherent in the particular weather situation. A simple method might involve selecting combinations of initial condition and stochastic perturbations to maximise spread in an ensem- ble of limited size, although more sophisticated measures of forecast uncertainty could also be used. For example, our results suggest that in weakly-forced weather patterns with widespread convection, it would be wise to make sure many stochastic realizations are available. Conversely, in stronglyforced weather patterns with little convection, the use of the stochastic scheme should not be given top priority. An important pre-requisite for such developments is be identification of an appropriate quantitative measure of large-scale forcing, such as the quasi-geostrophic omega equation diagnostic of Deveson et al. (2002).

Acknowledgements. This work has been carried out within the PANDOWAE Research Group FOR896 of the DFG (Deutsche Forschungsgemeinschaft). We thank the ECMWF for the computing resources made available for this work within the Special Project "Ensemble modelling for improvement of short-range precipitation forecasts". The German Weather Service DWD is gratefully acknowledged for providing radar data. On a personal note, the authors would like to thank Christian Keil for his help on working with the ECMWF Ensemble Prediction System, and Axel Seifert and Ulrich Schättler (DWD) for their assistance with running the COSMO model. We want to thank Tobias Selz and Richard Keane for their careful reading of the initial manuscript, that resulted in the correction of a few minor errors. Finally, we are thankful for the comments of two anonymous reviewers and of editor Peter Haynes which have helped to improve the manuscript.

Edited by: P. Haynes

\section{References}

Applequist, S., Gahrs, G. E., Pfeffer, R. L., and Niu, X.-F.: Comparison of Methodologies for Probabilistic Quantitative Precipitation Forecasting, Weather Forecast., 17, 783-799, 2002.

Berner, J., Doblas-Reyes, F. J., Palmer, T. N., Shutts, G. J., and Weisheimer, A.: Impact of a quasi-stochastic cellular automaton backscatter scheme on the systematic error and seasonal predicition skill of a global climate model, Phil. Trans. R. Soc A, 366, 2561-2579, doi:10.1098/rsta.2008.0031, 2008.

Berner, J., Shutts, G. J., Leutbecher, M., and Palmer, T. N.: A spectral stochastic kinetic energy backscatter scheme and its impact on flow-dependent predictability in the ECMWF Ensemble Prediction System. J. Atmos. Sci., 66, 603-626. doi:10.1175/2008JAS2677.1, 2009.

Bowler, N. E., Arribas, A., Beare, S. E., Mylne, K. R., and Shutts, G. J.: The local ETKF and SKEB: Upgrades to the MOGREPS short-range ensemble prediction system, Q. J. Roy. Meteorol. Soc., 135, 767-776, 2009.

Bremnes, J. B.: Probabilistic Forecasts of Precipitation in Terms of Quantiles Using NWP Model Output, Mon. Weather Rev., 132, 338-347, 2003.

Bretherton, C. S. and Smolarkiewicz, P. K.: Gravity Waves, Compensation Subsidence and Detrainment around Cumulus Clouds, J. Atm. Sci., 46, 740-759, 1989. 
Bright, D. R. and Mullen, S. L.: Short-range ensemble forecasts of precipitation during the southwest monsoon, Weather Forecast., 17, 1080-1100, 2002.

Buizza, R. and Palmer, T. N.: The singular vector structure of the atmospheric general circulation, J. Atmos. Sci., 52, 1434-1456, 1995.

Buizza, R., Miller, M., and Palmer, T. N.: Stochastic representation of model uncertainties in the ECMWF Ensemble Prediction System, Q. J. Roy. Meteorol. Soc., 125, 2887-2908, 1999.

Buizza, R., Houtekamer, P. L., Toth, Z., Pellerin, G., Wei, M., and Zhu, Y.: A comparison of the ECMWF, MSC, and NCEP Global Ensemble Prediction Systems, Mon. Weather Rev., 133, 10761097, 2005.

Cohen, B. G.: Fluctuations in an ensemble of cumulus clouds. Ph.D. thesis, University of Reading, 2001.

Corsmeier, U., Kalthoff, N., Barthlott, C., Aoshima, F., Behrendt, A., Di Girolamo, P., Dorninger, M., Handwerker, J., Kottmeier, C., Mahlke, H., Mobbs, S., Norton, E., Wickert, J., and Wulfmeyer, V.: Processes driving deep convection over complex terrain: a multi-scale analysis of observations from COPS IOP 9c, Q. J. Roy. Meteorol. Soc., 137, 137-155.

Craig, G. C. and Cohen, B. G.: Fluctuations in an equilibrium convective ensemble. Part I: Theoretical formulation, J. Atmos. Sci., 63, 1996-2004, 2006.

Deveson, A. C. L., Browning, K. A., and Hewson, T. D.: A classification of FASTEX cyclones using a height-attributable quasigeostrophic vertical-motion diagnostic, Q. J. Roy. Meteorol. Soc., 128, 93-117, 2002.

DWD: Description of the COSMO-EU setup of the COSMO model, available at: http://www.cosmo-model.org/content/tasks/ operational/dwd/default_eu.htm, 7 April 2011

Grams, C. N., Wernli, H., Bottcher, M., Čampa, J., Corsmeier, U., Jones, S. C., Keller, J. H., Lenz, C.-J., and Wiegand, L.: From the extratropical transition of Hanna (2008) to a Mediterranean cyclone: the key role of diabatic processes, Q. J. Roy. Meteor. Soc., submitted, 2011.

Groenemeijer, P., and Coauthors: Observations of Kinematics and Thermodynamic Structure Surrounding a Convective Storm Cluster over a Low Mountain Range. Mon. Wea. Rev., 137, 585602, 2009.

Houtekamer, P. L. and Derome, J.: Methods for ensemble prediction. Mon. Weather Rev., 123, 2181-2196, 1995.

Kain, J. S.: The Kain-Fritsch convective parameterization: An update, J. Appl. Meteor., 43, 170-181, 2004.

Kain, J. S. and Fritsch, J. M.: A one-dimensional entraining/detraining plume model and its application in convective parameterization, J. Atmos. Sci., 47, 2784-2802, 1990.

Khouider, B., Majda A. J., and Katsoulakis M. A.: Coarse-grained stochastic models for tropical convection and climate. Proc. Natl. Acad. Sci., 100, 11941-11946, 2003.

Lorenz, E. N.: Deterministic Nonperiodic Flow, J. Atmos. Sci., 20, 130-141, 1963.

Molteni, F., Buizza, R., Palmer, T. N., and Petroliagis, T.: The ECMWF ensemble prediction system: methodology and validation, Q. J. Roy. Meteorol. Soc., 122, 73-119, 1996.
Molteni, F., Buizza, R., Marsigli, C., Montani, A., Nerozzi, F., and Paccagnella, T.: A strategy for High-Resolution Ensemble Prediction, Part I: Definition of Representative Members and Global Model Experiments, Q. J. Roy. Meteor. Soc., 127, 2069-2094, 2001.

Palmer, T. N.: A nonlinear dynamical perspective on model error: A proposal for non-local stochastic dynamic parametrization in weather and climate prediction models. Q. J. Roy. Meteor. Soc., 127, 279-304, 2001.

Plant, R. S. and Craig, G. C.: A stochastic parameterization for deep convection based on equilibrium statistics, J. Atmos. Sci., 65, 87-105, 2008.

Púčik, T., Francová, M., Rýva, D., Kolář, M., and Ronge, L.: Forecasting challenges during the severe weather outbreak in Central Europe on 25 June 2008, Atmos. Res, 100, 680-704, 2011.

Reynolds, C. A., Teixeira, J., and McLay, J. G.: Impact of Stochastic Convection on the Ensemble Transform, Mon. Weather Rev., 136, 4517-4526, 2008.

Schättler, U., Doms, G., and Schraff, C.: A description of the nonhydrostatic regional COSMO-model, part VII: user's guide, http: //www.cosmo-model.org, last access: 7 April 2011.

Shutts, G.: A kinetic energy backscatter algorithm for use in ensemble prediction system, Q. J. Roy. Meteor. Soc., 131, 3079-3102, doi: 10.1256/qj.04.106, 2005.

Simon A., Kaňák, J., Sokol, A., Putsay, M., Uhrínová, L., Csirmaz, K., Okon, L., and Habrovský, R.: Case study of a severe windstorm over Slovakia and Hungary on 25 June 2008, Atmos. Res., 100, 705-739, 2011.

Stensrud, D. J., Bao, J.-W., and Warner, T. T.: Using Initial Condition and Model Physics Perturbations in Short-Range Ensemble Simulations of Mesoscale Convective Systems. Mon. Weather Rev., 128, 2077-2107, 2000.

Tan, Z.-M., Zhang, F., Rotunno, R., and Snyder, C.: Mesoscale Predictability of Moist Baroclinic Waves: Experiments with Parameterized Convection, J. Atmos. Sci., 61, 1794-1804, 2004.

Teixeira, J. and Reynolds, C. A.: Stochastic Nature of Physical Parameterizations in Ensemble Prediction: A Stochastic Convection Approach, Mon. Weather Rev., 136, 483-496, 2008.

Tennant, W. J., Shutts, G. J., Arribas, A., and Thompson, S. A.: Using a stochastic kinetic energy backscatter scheme to improve MOGREPS probabilistic forecast skill. Mon. Wea. Rev., 139, 1190-1206, doi:10.1175/2010MWR3430.1, 2011.

Theis, S. E., Hense, A., and Damrath, U.: Probabilistic precipitation forecasts from a deterministic model: a pragmatic approach, Meteorol. Appl., 12, 257-268, 2005.

Toth, Z. and Kalnay, E.: Ensemble forecasting at NMC: The generation of perturbations, B. Am. Meteorol. Soc., 74, 2317-2330, 1993.

Toth, Z. and Kalnay, E.: Ensemble forecasting at NCEP and the breeding method. Mon. Wea. Rev., 125, 3297-3319, 1997.

Zhang, F., Bei, N., Rotunno, R., Snyder, C., and Epifano, C.: Mesoscale Predictability of Moist Baroclinic Waves: Convection-Permitting and Multistage Error Growth Dynamics, J. Atmos. Sci., 64, 3579-3594, 2007. 